\title{
A COMPARATIVE STUDY ON THE EFFICIENCY OF TEST STATISTICS IN TESTING THE DIFFERENCES BETWEEN TWO DEPENDENT DATASETS
}

\author{
Unchalee Tonggumnead \\ Department of Mathematics and Computer Science \\ Rajamangala University of Technology Thanyaburi \\ THAILAND
}

\begin{abstract}
The purpose of the present study is to compare the efficiency of test statistics in testing the differences between two dependent datasets applying a regression framework with centered independent variable values. Comparisons of the efficiency of the test statistics, namely the $t_{b_{0}^{*}}^{R C M}$, the Wilcoxon matched-pairs signed-ranks test, and the Paired sample t-test, are made with the correlation coefficients, the sample sizes, and the ratio of mean different between the two datasets being varied. Simulations of the test statistics apply a Monte Carlo technique and are repeated 1,000 times. The research results show that the efficiency in controlling Type I errors of the Wilcoxon matched-pairs signed-ranks test and the Paired t-test is high under all scenarios, while that of the $t_{b_{0}^{*}}^{R C M}$ is high only in case the $r_{x y}$ is not excessively high, that is, under 0.80. In contrast, the power of the test of the latter is significantly higher than the former under all scenarios.
\end{abstract}

AMS Subject Classification: 62F03, 62F 30

Key Words: paired sample t-test; Wilcoxon matched-pairs signed-ranks test; regression analysis; center model

\section{Introduction}

The primary purpose of a comparison of the mean difference between two inReceived: June 30, 2020 (C) 2021 Academic Publications 
dependent datasets, such as the mean difference of the dependent variable of a control group and an experimental group, is to identify whether the independent variable differently affects the dependent variable. This can be conducted using two methods: a t-test and a regression analysis. For the t-test, the two datasets under investigation need to comprise an independent variable in the form of categorical, discrete data and a dependent variable in the form of quantitative, continuous data. For the regression analysis, the independent variable of the control group is assigned a dummy value of 0 , and the independent variable of the experimental group a dummy value of 1 . A product of this type of analysis is a regression equation with the value of the $y$-intercept equaling the mean of the dependent variable of the control group and the regression coefficient, i.e. the slope, equaling the rate of the change in the dependent variable $y$ resulting from a 1 -unit change in the independent variable $x$. It can thus be concluded that in comparing the mean difference between two independent datasets, the t-test and the regression analysis are along similar lines [9]. In the case of homogeneity of variance, the t-test can take the form of an analysis of variance, in which the independent variable serves as the treatment variable and the extraneous variable has to be controlled in order to prevent its effects on the dependent variable [10]. In research involving a comparison of the mean difference between two datasets that are dependent or bear a one-to-one relationship, the data for each of the corresponding pairs have to be collected under the same condition. It important that the data be treated in a stringent manner not only to ensure the similarities within each of the corresponding pairs but also to highlight the differences across pairs. Non-parametric test statistics for such a purpose include the Wilcoxon matched-pairs signed-ranks test proposed by Wilcoxon in 1949 [6] and [14], while parametric ones include the Paired sample t-test, sometimes called the dependent t-test. In the Paired sample t-test, an analysis is performed to determine whether the mean difference between two datasets equals 0. Additionally, each of the subjects or entities is measured twice in paired observations. One common application of the Paired sample t-test is a case-control study using a repeated measures design, especially a pre-test, post-test design, in which the intervention is assumed to lead to an outcome measure improvement in a causal manner. One drawback of this approach of evaluation is that a counterfactual inference drawn from it may be weak [15],[3] and [5] due to the effect of a confounding variable, such as history, maturation, instrumentation, subject selection, and test effects, on the change in a dependent variable. However, confounding variables do not constitute the emphases of the present study. Additionally, such variables can be dealt with using certain measures. For instance, those difficult to control may 
be regarded as independent variables that also need to be examined. Alternatively, confounding variables may be controlled statistically using an analysis of covariance to lessen the differences between the two datasets and ensure that only the experiment results in an outcome measure improvement or change [2]. Therefore, the present study aims to compare the efficiency of three test statistics, namely the $t_{b_{0}^{*}}^{R C M}$, the Wilcoxon matched-pairs signed-ranks test, the Paired sample t-test, in testing the differences between two dependent datasets applying a regression framework with centered independent variable values [4]. The correlation coefficients, the sample sizes, the ratio of mean different and the ration of variance between the two data sets are varied. The ultimate objective of this research is to assess the weakness inherent in the pre-test, post-test design associated with the Paired sample t-test under the assumption that the background knowledge of the subjects, manifesting itself in the form of pre-test scores, has a significant effect on the mean difference between the two datasets.

\section{Methodology}

\subsection{The classical Paired sample t-test}

The Paired sample t-test, sometimes called the dependent sample t-test, is a statistical procedure used to determine whether the mean difference between two sets of observations is zero. In a paired sample t-test, each subject or entity is measured twice, resulting in pairs of observations. Common applications of the paired sample t-test include pretest-posttest designs. The relationship between the variables is written as

$$
y_{i}=x_{i}+d_{i}
$$

where $x_{i}$ is the pre-test, $y_{i}$ is the post-test, $d_{i}=y_{i}-x_{i}$ is the difference between post-test $\left(y_{i}\right)$ and pre-test $\left(x_{i}\right)$, which $x_{i}, y_{i}, d_{i}$ are normal distribution $x \sim$ $N\left(\mu_{x}, \sigma_{x}\right), y \sim N\left(\mu_{y}, \sigma_{y}\right)$ and $d \sim N\left(\mu_{d}, \sigma_{d}\right)$ respectively. We know $d_{i}=y_{i}-x_{i}$, so that

$$
\sigma_{d}^{2}=\sigma_{y}^{2}+\sigma_{x}^{2}-2 \operatorname{Cov}(x, y)
$$

Since, $\operatorname{Cov}(x, y)=\rho_{x y} \sqrt{\sigma_{x}^{2} \sigma_{y}^{2}}$, so that

$$
\sigma_{d}^{2}=\sigma_{y}^{2}+\sigma_{x}^{2}-2 \rho_{x y} \sigma_{x} \sigma_{y}
$$


At the same time $\sigma_{\bar{d}}^{2}=\frac{\sigma_{d}^{2}}{n}$, so that

$$
\sigma_{\bar{d}}^{2}=\frac{\sigma_{y}^{2}+\sigma_{x}^{2}-2 \rho_{x y} \sigma_{x} \sigma_{y}}{n}
$$

or

$$
\sigma_{\bar{d}}=\sqrt{\frac{\sigma_{y}^{2}+\sigma_{x}^{2}-2 \rho_{x y} \sigma_{x} \sigma_{y}}{n}}
$$

\subsection{Simple linear regression and center model}

In the regression analysis, the simple linear regression model is given by

$$
y_{i}=\beta_{0}+\beta_{1} x_{i}+\varepsilon_{i},
$$

where $y_{i}$ is dependent variable, $x_{i}$ is independent variable, $\beta_{0}, \beta_{1}$ are unknown parameter or regression coefficient, $\varepsilon_{i}$ is random error [11]. In some cases, the model (6) is very useful when measuring the independent variable around its mean $\left(x_{i}-\bar{x}\right)$. The model in equation (6) is written as

$$
\begin{array}{r}
y_{i}=\beta_{0}+\beta_{1}\left(x_{i}-\bar{x}\right)+\beta_{1} \bar{x}+\varepsilon_{i}, \\
y_{i}=\beta_{0}^{*}+\beta_{1}\left(x_{i}-\bar{x}\right)+\varepsilon_{i}, \text { where } \beta_{0}^{*}=\beta_{0}+\beta_{1} \bar{x} .
\end{array}
$$

The sum of square of error is given by

$$
S\left(\beta_{0}^{*}, \beta_{1}\right)=\sum_{1}^{n} e_{i}^{2}=\sum_{i=1}^{n}\left(y_{i}-\beta_{0}^{*}-\beta_{1}\left(x_{i}-\bar{x}\right)\right)^{2} .
$$

To minimize $S\left(\beta_{0}^{*}, \beta_{1}\right)$, this expression differentiated with respect to $\beta_{0}^{*}$ and $\beta_{1}$, the derivative is set equal to zero $\frac{\partial S\left(\beta_{0}^{*}, \beta_{1}\right)}{\partial \beta_{0}^{*}}=0$ and $\frac{\partial S\left(\beta_{0}^{*}, \beta_{1}\right)}{\partial \beta_{1}}=0$, the least square estimator of $\beta_{0}^{*}$ and $\beta_{1}$ are $b_{0}^{*}=\bar{y}$ and $b_{1}=\frac{S_{x y}}{S_{x x}}$, under the assumption that $E\left(\varepsilon_{i}\right)=0, \operatorname{Var}\left(\varepsilon_{i}\right)=\sigma^{2}, \operatorname{Cov}\left(\varepsilon_{i}, \varepsilon_{j}\right)=0, E\left(b_{0}^{*}\right)=\beta_{0}^{*}, E\left(b_{1}\right)=\beta_{1}$, $\operatorname{Var}\left(b_{0}^{*}\right)=\frac{\sigma^{2}}{n}, \operatorname{Var}\left(b_{1}\right)=\frac{\sigma^{2}}{S_{x x}}$. Similarly, if the model is in the form of paired sample t-test that the subject or the entity is measured twice, where $y_{i}=x_{i}+d_{i}$ or $d_{i}=y_{i}-x_{i}$. Let $d_{i}=y_{i}-x_{i}$ be the dependent variable, and the independent variables that affect to $d_{i}$ is $x_{i}-\bar{x}$. So, the regression model is

$$
d_{i}=\beta_{0}^{*}+\beta_{1}\left(x_{i}-\bar{x}\right)+\varepsilon_{i}
$$


The least square estimator of $\beta_{0}^{*}$ is $b_{0}^{*}=\bar{d}[8],[12]$ and [1]. Therefore, the mean different tests between the two related groups are equal to 0 is testing $\beta_{0}^{*}=0$ or testing intercept $=0$. The test statistic is in the form of $t_{b_{0}^{*}}^{R C M}=\frac{b_{0}^{*}}{\sigma_{b_{0}}^{*}}$, where RCM represent the Regression Center Model, $\sigma_{b_{0}^{*}}^{2}=\frac{1}{n} \times \frac{S S E}{n-2}$. From [4], the SSE will be smaller than the case of regression that does not consider the covariate. Because replace the error $e_{i}=\left(y_{i}-x_{i}\right)-(\bar{y}-\bar{x})$ with $e_{i}=\left(y_{i}-\bar{x}\right)-\left(\bar{d}+\beta_{1}\left(x_{i}-\bar{x}\right)\right)$, and the variance of any predicted value without the term involving the deviation from mean of $x$ since the mean of $x$ is 0 . This is written as

$$
\begin{aligned}
& \sigma_{b_{0}^{*}}^{2}=\frac{1}{n}\left(\frac{S S E}{n-2}\right), \\
& \sigma_{b_{0}^{*}}^{2}=\frac{1}{n}\left(\frac{\sum_{i=1}^{n}\left[\left(y_{i}-\bar{x}\right)-(\bar{y}-\bar{x})-\beta_{1}\left(x_{i}-\bar{x}\right)\right]^{2}}{n-2}\right), \\
& \sigma_{b_{0}^{*}}^{2}=\frac{1}{n}\left(\frac{\sum_{i=1}^{n}\left[\left(y_{i}-\bar{y}\right)-\beta_{1}\left(x_{i}-\bar{x}\right)\right]^{2}}{n-2}\right), \\
& \sigma_{b_{0}^{*}}^{2}=\frac{1}{(n)(n-2)}\left(\sum_{i=1}^{n}\left[\left(y_{i}-\bar{y}\right)^{2}-2\left(y_{i}-\bar{y}\right) \beta_{1}\left(x_{i}-\bar{x}\right)+\beta_{1}^{2}\left(x_{i}-\bar{x}\right)^{2}\right]\right), \\
& \sigma_{b_{0}^{*}}^{2}=\frac{1}{(n)(n-2)}\left(\sum _ { i = 1 } ^ { n } \left[y_{i}^{2}-2 y_{i} \bar{y}+\bar{y}^{2}-2 y_{i} \beta_{1}\left(x_{i}-\bar{x}\right)+2 \bar{y} \beta_{1}\left(x_{i}-\bar{x}\right)\right.\right. \\
& \left.\left.+\beta_{1}^{2}\left(x_{i}^{2}-2 x_{i} \bar{x}+\bar{x}^{2}\right)\right]\right), \\
& \sigma_{b_{0}^{*}}^{2}=\frac{1}{(n)(n-2)}\left(\sum_{i=1}^{n} y_{i}^{2}-2 \sum_{i=1}^{n} y_{i} \bar{y}+\sum_{i=1}^{n} \bar{y}^{2}-2 \beta_{1} \sum_{i=1}^{n} x_{i} y_{i}\right. \\
& +2 \beta_{1} \sum_{i=1}^{n} y_{i} \bar{x}+2 \beta_{1} \sum_{i=1}^{n} x_{i} \bar{y}-2 \beta_{1} \sum_{i=1}^{n} \bar{x} \bar{y}+\beta_{1}^{2} \sum_{i=1}^{n} x_{i}^{2} \\
& \left.-2 \beta_{1}^{2} \sum_{i=1}^{n} x_{i} \bar{x}+\beta_{1}^{2} \sum_{i=1}^{n} \bar{x}^{2}\right) \\
& \sigma_{b_{0}^{*}}^{2}=\frac{1}{(n)(n-2)}\left(\sum_{i=1}^{n} y_{i}^{2}-2 n \bar{y}^{2}+n \bar{y}^{2}-2 \beta_{1} \sum_{i=1}^{n} x_{i} y_{i}\right. \\
& \left.+2 n \bar{y} \beta_{1} \bar{x}+2 n \bar{y} \beta_{1} \bar{x}-2 n \bar{y} \beta_{1} \bar{x}+\beta_{1}^{2} \sum_{i=1}^{n} x_{i}^{2}-2 \beta_{1}^{2} n \bar{x}^{2}+\beta_{1}^{2} \sum_{i=1}^{n} \bar{x}^{2}\right),
\end{aligned}
$$




$$
\sigma_{b_{0}^{*}}^{2}=\frac{1}{(n)(n-2)}\left(\sum_{i=1}^{n} y_{i}^{2}-n \bar{y}^{2}-2 \beta_{1} \sum_{i=1}^{n} x_{i} y_{i}+\beta_{1}^{2} \sum_{i=1}^{n} x_{i}^{2}\right) .
$$

In the term of population, we can rewrite the $\sigma_{b_{0}^{*}}^{2}$ as

$$
\begin{gathered}
\sigma_{b_{0}^{*}}^{2}=\frac{1}{(n)(n)}\left(\sum_{i=1}^{n} y_{i}^{2}-n \bar{y}^{2}-2 \beta_{1} \sum_{i=1}^{n} x_{i} y_{i}+\beta_{1}^{2} \sum_{i=1}^{n} x_{i}^{2}\right), \\
\sigma_{b_{0}^{*}}^{2}=\frac{1}{(n)}\left(\frac{\sum_{i=1}^{n} y_{i}^{2}}{n}-\bar{y}^{2}-\frac{2 \beta_{1} \sum_{i=1}^{n} x_{i} y_{i}}{n}+\frac{\beta_{1}^{2} \sum_{i=1}^{n} x_{i}^{2}}{n}\right) .
\end{gathered}
$$

Which can be reformatted into $\frac{\sum_{i=1}^{n} y_{i}^{2}}{n}-\bar{y}^{2}$ is variance of $y$, and at the same time, we consider at $\bar{x}=0, \frac{\sum_{i=1}^{n} x_{i}^{2}}{n}$ is a variance of $x$, and $\frac{\sum_{i=1}^{n} x_{i} y_{i}}{n}$ is a $\operatorname{Cov}\left(x_{i}, y_{i}\right)$. So, we can rewrite the $\sigma_{b_{0}^{*}}^{2}$ as

$$
\begin{gathered}
\sigma_{b_{0}^{*}}^{2}=\frac{1}{n}\left(\sigma_{y}^{2}-2 \beta_{1} \operatorname{Cov}\left(x_{i}, y_{i}\right)+\beta_{1}^{2} \sigma_{x}^{2}\right), \\
\sigma_{b_{0}^{*}}^{2}=\frac{1}{n}\left(\sigma_{y}^{2}-2 \beta_{1} \beta_{1} \sigma_{x}^{2}+\beta_{1}^{2} \sigma_{x}^{2}\right), \\
\sigma_{b_{0}^{*}}^{2}=\frac{1}{n}\left(\sigma_{y}^{2}-2 \beta_{1}^{2} \sigma_{x}^{2}+\beta_{1}^{2} \sigma_{x}^{2}\right), \\
\sigma_{b_{0}^{*}}^{2}=\frac{1}{n}\left(\sigma_{y}^{2}-\beta_{1}^{2} \sigma_{x}^{2}\right), \\
\sigma_{b_{0}^{*}}^{2}=\frac{1}{n}\left(\sigma_{y}^{2}-\left(\rho_{x y} \frac{\sigma_{y}}{\sigma_{x}}\right)^{2} \sigma_{x}^{2}\right), \\
\sigma_{b_{0}^{*}}^{2}=\frac{1}{n}\left(\sigma_{y}^{2}-\rho_{x y}^{2} \sigma_{y}^{2}\right) .
\end{gathered}
$$

So, the variance of $b_{0}^{*}$ is

$$
\sigma_{b_{0}^{*}}^{2}=\frac{1}{n}\left(\sigma_{y}^{2}-\rho_{x y}^{2} \sigma_{y}^{2}\right)
$$




\subsection{Comparing the standard deviation of $\bar{d}$ or $\sigma_{\bar{d}}$ in the case of Paired sample t-test and $\sigma_{b_{0}^{*}}$ of regression center model}

Let $\sigma_{x}^{2}=10$ and $\sigma_{y}^{2}=40$, and let $r_{x y}$ be varied from 0.05 to 1.00 and $\mathrm{n}$ be varied from 5 to 100 . When the $\sigma_{b_{0}^{*}}$ of regression center model, as shown in Fig. 1(a), and the $\sigma_{\bar{d}}$ of Paired sample t-test, as shown in Fig. 1(b), it is found that the value of the $\sigma_{b_{0}^{*}}$ is lower than that of the Paired sample t-test. In addition, $\sigma_{b_{0}^{*}}$ when the values of $\mathrm{n}$ and $r_{x y}$ rise, the values of the $\sigma_{b_{0}^{*}}$ of regression center model and the $\sigma_{\bar{d}}$ of Paired sample t-test decline. Also, when the ratio between the $\sigma_{b_{0}^{*}}$ and the $\sigma_{\bar{d}}$, as shown in Fig. 1(c), is taken into account, it is found that $\frac{\sigma_{b_{0}^{*}}}{\sigma_{\bar{d}}}<1$ under almost all scenarios. Thus, the test statistic for testing the mean different between two dependent datasets is $t=\frac{\bar{d}}{\sqrt{\frac{\sigma_{y}^{2}+\sigma_{x}^{2}-2 \rho_{x y} \sigma_{x} \sigma_{y}}{n}}}$ in the Paired sample t-test and testing intercept $\beta_{0}^{*}=0$ in regression center model, where the test statistic can be represented as $t_{b_{0}^{*}}^{R C M}=\frac{\bar{d}}{\sqrt{\frac{\left(\sigma_{y}^{2}-\rho_{x y}^{2} \sigma_{y}^{2}\right)}{n}}}$. One principle in constructing a test statistic is the effect of the variance of an estimator on its efficiency. In case the variance of the estimator is excessively low, the value of the test statistic will become excessively high, increasing the chance of hypothesis rejection. The next section will detail the ability to control Type I error and the power of the test of the $t_{b_{0}^{*}}^{R C M}$, the Wilcoxon matched-pairs signed-ranks test, and the Paired sample t-test under different scenarios. 


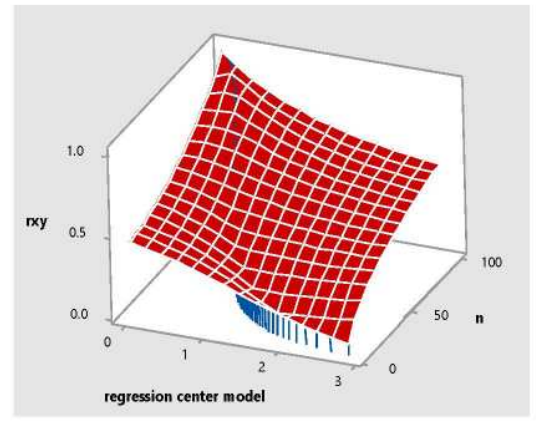

(a)

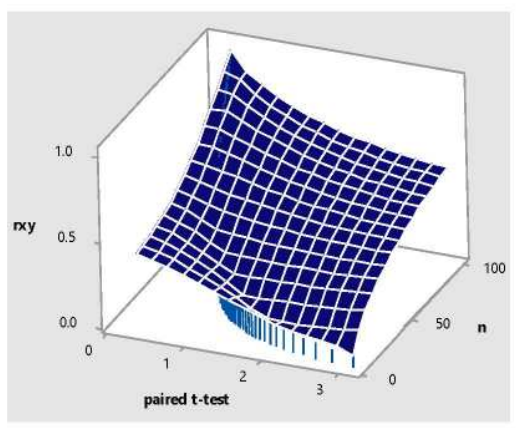

(b)

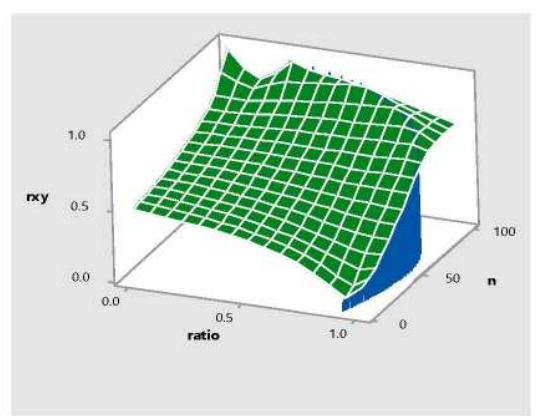

(c)

Figure 1: (a) Illustrate $\sigma_{b_{0}^{*}}$ of regression center model when $r_{x y}$ be varied from 0.05 to 1.00 and $\mathrm{n}$ be varied from 5 to 100. (b) Illustrate $\sigma_{\bar{d}}$ of Paired sample t-test when $r_{x y}$ be varied from 0.05 to 1.00 and $\mathrm{n}$ be varied from 5 to 100 . (c) Illustrate the ratio of $\frac{\sigma_{b_{0}^{*}}}{\sigma_{\bar{d}}}$ when $r_{x y}$ be varied from 0.05 to 1.00 and $n$ be varied from 5 to 100 .

\section{Simulation study}

The purpose of this study is to compare the performance of three test statistics (Wilcoxon matched pairs signed-ranks test, Paired sample t-test, $t_{b_{0}^{*}}^{R C M}$ ) for comparing the differences between 2 groups of data that are related or not independent from one another. The ability for control Type I error and power the test are considered. The procedures of simulation study are as follows:

1. Data distribution patterns: $X$ is the first data set or pre-test: $Y$ is the second data set or post-test, $X$ and $Y$ are related at different levels, and $r_{x y}$ be varied from 0.05 to 1.00 . The procedure for generating variable are as follows:

1.1. Generate $X_{1}$ and $X_{2}$ from the standard normal distribution.

1.2. Let $X_{3}$ be a linear combination of $X_{1}$ and $X_{2}$ by $X_{3}=r_{x y} X_{1}+$ 
$\sqrt{\left(1-r_{x y}^{2}\right)} X_{2}$

1.3. Let $X=\mu_{1}+\sigma_{1} X_{1}$ and $Y=\mu_{2}+\sigma_{2} X_{3}$, so that now $X$ and $Y$ have a correlation $r_{x y}[13]$.

2. Under the null hypothesis $\left(H_{0}\right)$ when it is true, let $\mu_{1}=\mu_{2}=10, \sigma_{1}^{2}=10$ and $\sigma_{2}^{2}=40$.

3. When the alternative hypothesis $\left(H_{1}\right)$ true, let $\mu_{2}=r \mu_{1}$, where $\mu_{1}=10$, $\mathrm{r}=1.3$ and $\mathrm{r}=1.5, \sigma_{1}^{2}=10$ and $\sigma_{2}^{2}=40$.

4. Let the sample sizes $n=10,20,30,40$ and 50 .

5. Calculate three test statistics values and the probabilities of Type I error, i.e. rejecting the null hypothesis $\left(H_{0}\right)$ when it is true, and the power of the test, i.e. rejecting $\left(H_{0}\right)$ when it is false, are calculated from 1,000 replications.

6. The control of Type I error employs the test statistic $Z$ in conducting a two-tailed test [7], where $\alpha$ represents the occurrence of Type I error, $\hat{\alpha}$ represents the estimated value of the occurrence of Type I error, $\alpha_{0}$ represents Type I error determined for this research, i.e. $\alpha=0.01$ and $\alpha=0.05, \mathrm{n}$ represents the number of replications determined for the present study, i.e. 1,000 .

The test hypotheses are $H_{0}: \alpha=\alpha_{0}$ and $H_{1}: \alpha \neq \alpha_{0}$, and the test statistic is $Z=\frac{\hat{\alpha}-\alpha_{0}}{\sqrt{\frac{\alpha_{0}\left(1-\alpha_{0}\right)}{n}}}$. Therefore, if the estimated value of the occurrence of Type I error $\hat{\alpha} \in\left[\alpha_{0}-Z_{\alpha / 2} \sqrt{\frac{\alpha_{0}\left(1-\alpha_{0}\right)}{n}}, \alpha_{0}+Z_{\alpha / 2} \sqrt{\frac{\alpha_{0}\left(1-\alpha_{0}\right)}{n}}\right]$, it can be assumed to be able to control this type of error. The finding indicates that the control ranges of Type I error when $\alpha=0.01$ and $\alpha=0.05$ are [0.002,0.018] and [0.036, 0.063], respectively.

\section{Results}

A comparison of the ability to control Type I errors of the Wilcoxon matchedpairs signed-ranks test, the Paired sample t-test, and the $t_{b_{0}^{*}}^{R C M}$ shows that the first two are efficient under all scenarios, while the latter does not perform well in case the value of $r_{x y}$ is excessively high. Specifically, the ability of the $t_{b_{0}^{*}}^{R C M}$ to control Type I errors deteriorates when $n=10,20,30,40$, and 50 at the significance level of $\alpha=0.01$ in case the value of reaches $0.90,0.85,0.85,0.80$, and 0.80 , respectively. In a similar vein, its ability to control Type I errors falls when $n=10,20,30,40$, and 50 at the significance level of $\alpha=0.05$ in case the value of $r_{x y}$ hits $0.90,0.85,0.85,0.80$, and 0.80 , respectively. The findings are displayed in Tables 1-5 and Fig. 2-3. 
In contrast, a comparison of the power of the test of the Wilcoxon matchedpairs signed-ranks test, the Paired sample t-test, and the $t_{b_{0}^{*}}^{R C M}$ demonstrates an opposite tendency. That is, the power of the test of the $t_{b_{0}^{*}}^{R C M}$ is distinctively the highest at the significance level of $\alpha=0.01$ when $n=10,20,30,40$, and 50 in case the mean difference between the two datasets takes the form of $\mu_{y}=1.3 \mu_{x}$ and the value of $r_{x y}$ is varied from 0.10 to 0.95 , followed by that of the Paired sample t-test and the Wilcoxon matched-pairs signed-ranks test, respectively. A further analysis at the significance level of $\alpha=0.05$ also yields similar results. Additionally, the power of the test of all the three test statistics is higher in case the value of $r_{x y}$ and the sample sizes from the two datasets increase. The findings are illustrated in Tables 6-10. Similarly, the power of the test of the $t_{b_{0}^{*}}^{R C M}$ is still significantly higher than that of the Paired sample t-test and the Wilcoxon matched-pairs signed-ranks test at the significance level of both $\alpha=0.01$ and $\alpha=0.05$ when $n=10,20,30,40$, and 50 in case the mean difference between the two datasets takes the form of $\mu_{y}=1.5 \mu_{x}$ and the value of $r_{x y}$ is varied from 0.10 to 0.95 . Also, the power of the test of the $t_{b_{0}^{*}}^{R C M}$ reaches 1.000 across all the $r_{x y}$ values in case the sample sizes from the two datasets are 20 or over. The findings are shown in Tables 11-15 and Fig. 4-5. 


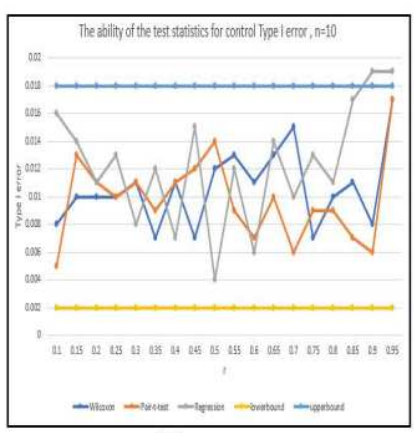

(a)

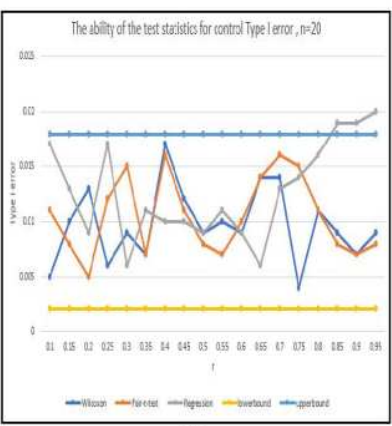

(b)



(c)

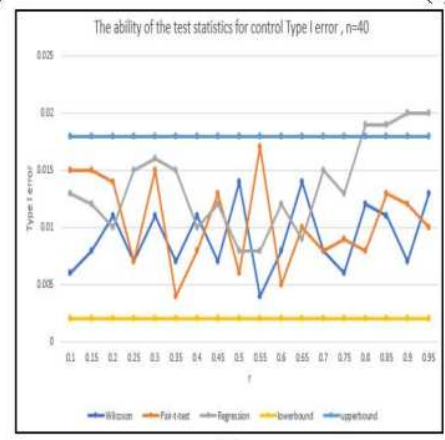

(d)

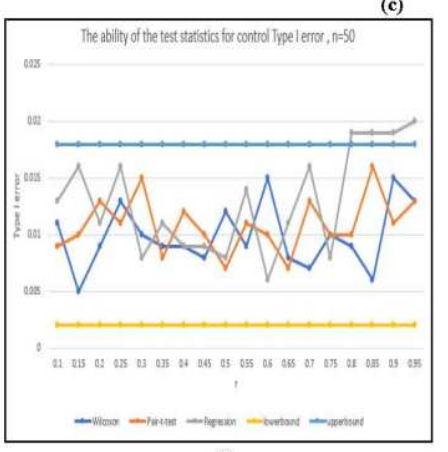

(e)

Figure 2: Illustrate the ability to control Type I error of the test statistics Wilcoxon matched pairs signed-ranks test, Paired sample $\mathrm{t}$-test, and $t_{b_{0}^{*}}^{R C M}, \alpha=0.01$ and $r_{x y}$ be varied from 0.10 to 0.95 : (a) $\mathrm{n}=10$, (b) $\mathrm{n}=20$, (c) $\mathrm{n}=30$, (d) $\mathrm{n}=40$ and (e) $\mathrm{n}=50$.

Table 1: Probabilities of rejection when $H_{0}$ is true $\left(\mu_{x}=\mu_{y}\right)$ of the test statistics Wilcoxon matched Pairs signed-ranks test, Paired sample t-test and $t_{b_{0}^{*}}^{R C M}$, sample size $\mathrm{n}=10, r_{x y}$ be varied from 0.10 to $0.95, \alpha=0.01$ and $\alpha=0.05$. 


\begin{tabular}{|c|c|c|c|c|c|c|c|}
\hline \multirow[t]{2}{*}{$\mathrm{n}$} & \multirow[t]{2}{*}{$r_{x y}$} & \multicolumn{3}{|c|}{$\alpha=0.01$} & \multicolumn{3}{|c|}{$\alpha=0.05$} \\
\hline & & $\overline{\mathrm{W}}$ & Paired & $t_{b_{0}^{*}}^{R C M}$ & $\overline{\mathrm{W}}$ & Paired & $t_{b_{0}^{*}}^{R C M}$ \\
\hline \multirow[t]{18}{*}{10} & 0.10 & 0.008 & 0.005 & 0.016 & 0.052 & 0.050 & 0.045 \\
\hline & 0.15 & 0.010 & 0.013 & 0.014 & 0.046 & 0.060 & 0.058 \\
\hline & 0.20 & 0.010 & 0.011 & 0.011 & 0.045 & 0.049 & 0.046 \\
\hline & 0.25 & 0.010 & 0.010 & 0.013 & 0.046 & 0.056 & 0.046 \\
\hline & 0.30 & 0.011 & 0.011 & 0.008 & 0.051 & 0.049 & 0.047 \\
\hline & 0.35 & 0.007 & 0.009 & 0.012 & 0.048 & 0.053 & 0.056 \\
\hline & 0.40 & 0.011 & 0.011 & 0.007 & 0.048 & 0.055 & 0.047 \\
\hline & 0.45 & 0.007 & 0.012 & 0.015 & 0.040 & 0.053 & 0.044 \\
\hline & 0.50 & 0.012 & 0.014 & 0.004 & 0.042 & 0.062 & 0.044 \\
\hline & 0.55 & 0.013 & 0.009 & 0.012 & 0.050 & 0.054 & 0.046 \\
\hline & 0.60 & 0.011 & 0.007 & 0.006 & 0.046 & 0.052 & 0.058 \\
\hline & 0.65 & 0.013 & 0.010 & 0.014 & 0.048 & 0.050 & 0.037 \\
\hline & 0.70 & 0.015 & 0.006 & 0.010 & 0.052 & 0.046 & 0.051 \\
\hline & 0.75 & 0.007 & 0.009 & 0.013 & 0.043 & 0.047 & 0.056 \\
\hline & 0.80 & 0.010 & 0.009 & 0.011 & 0.049 & 0.042 & 0.062 \\
\hline & 0.85 & 0.011 & 0.007 & 0.017 & 0.045 & 0.040 & $0.064^{*}$ \\
\hline & 0.90 & 0.008 & 0.006 & $0.019^{*}$ & 0.044 & 0.054 & $0.064^{*}$ \\
\hline & 0.95 & 0.017 & 0.017 & $0.019^{*}$ & 0.053 & 0.050 & $0.066^{*}$ \\
\hline
\end{tabular}



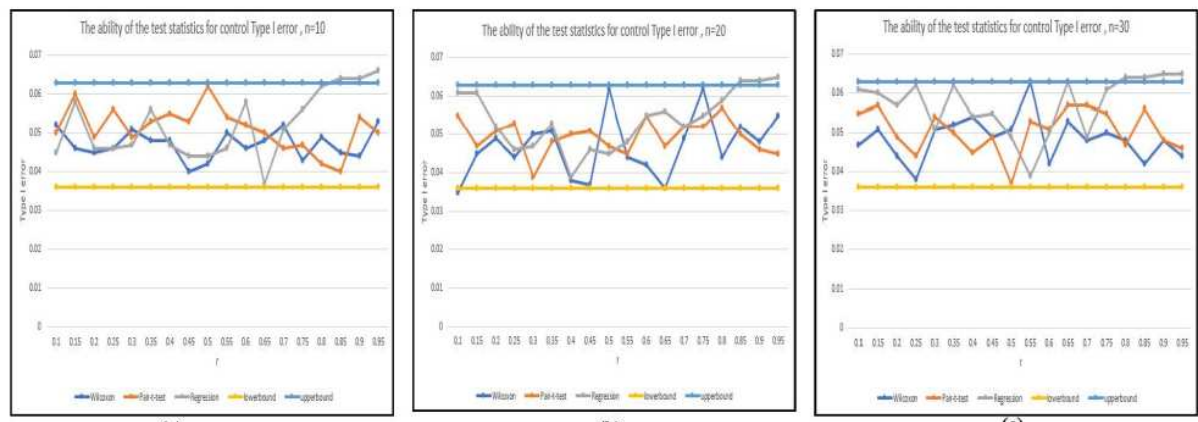

(a)

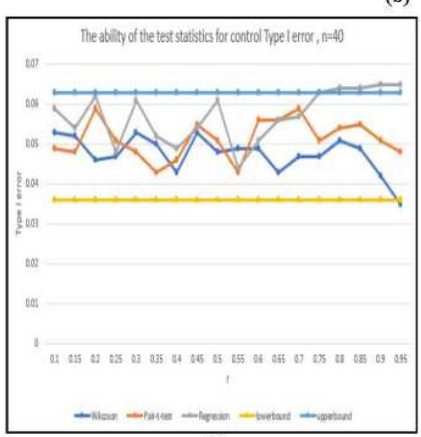

(d)

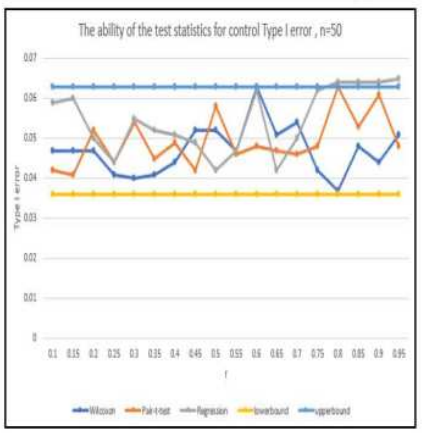

(e)

Figure 3: Illustrate the ability to control Type I error of the test statistics Wilcoxon matched pairs signed-ranks test, Paired sample $\mathrm{t}$-test , and $t_{b_{0}^{*}}^{R C M}, \alpha=0.05$ and $r_{x y}$ be varied from 0.10 to 0.95 : (a) $\mathrm{n}=10$, (b) $\mathrm{n}=20$, (c) $\mathrm{n}=30$, (d) $\mathrm{n}=40$ and (e) $\mathrm{n}=50$.. 
Table 2: Probabilities of rejection when $H_{0}$ is true $\left(\mu_{x}=\mu_{y}\right)$ of the test statistics Wilcoxon matched Pairs signed-ranks test, Paired sample t-test and $t_{b_{0}^{*}}^{R C M}$, sample size $\mathrm{n}=20, r_{x y}$ be varied from 0.10 to $0.95, \alpha=0.01$ and $\alpha=0.05$.

\begin{tabular}{|c|c|c|c|c|c|c|c|}
\hline $\mathrm{n}$ & $r_{x y}$ & \multicolumn{3}{|c|}{$\alpha=0.01$} & \multicolumn{3}{c|}{$\alpha=0.05$} \\
\cline { 3 - 8 } & & $\mathrm{W}$ & Paired & $t_{b_{0}^{*}}^{R C M}$ & $\mathrm{~W}$ & Paired & $t_{b_{0}^{*}}^{R C M}$ \\
\hline \multirow{4}{*}{20} & 0.10 & 0.005 & 0.011 & 0.017 & 0.035 & 0.055 & 0.061 \\
\cline { 2 - 8 } & 0.15 & 0.010 & 0.008 & 0.013 & 0.045 & 0.047 & 0.061 \\
\cline { 2 - 8 } & 0.20 & 0.013 & 0.005 & 0.009 & 0.049 & 0.051 & 0.052 \\
\cline { 2 - 8 } & 0.25 & 0.006 & 0.012 & 0.017 & 0.044 & 0.053 & 0.046 \\
\cline { 2 - 8 } & 0.30 & 0.009 & 0.015 & 0.006 & 0.050 & 0.039 & 0.047 \\
\cline { 2 - 8 } & 0.35 & 0.007 & 0.007 & 0.011 & 0.051 & 0.048 & 0.053 \\
\cline { 2 - 8 } & 0.40 & 0.017 & 0.016 & 0.010 & 0.038 & 0.050 & 0.039 \\
\cline { 2 - 8 } & 0.45 & 0.012 & 0.011 & 0.010 & 0.037 & 0.051 & 0.046 \\
\cline { 2 - 8 } & 0.50 & 0.009 & 0.008 & 0.009 & 0.062 & 0.047 & 0.045 \\
\cline { 2 - 8 } & 0.55 & 0.010 & 0.007 & 0.011 & 0.044 & 0.045 & 0.048 \\
\cline { 2 - 8 } & 0.60 & 0.009 & 0.010 & 0.009 & 0.042 & 0.055 & 0.055 \\
\cline { 2 - 8 } & 0.65 & 0.014 & 0.014 & 0.006 & 0.036 & 0.047 & 0.056 \\
\cline { 2 - 8 } & 0.70 & 0.014 & 0.016 & 0.013 & 0.049 & 0.052 & 0.052 \\
\cline { 2 - 8 } & 0.75 & 0.004 & 0.015 & 0.014 & 0.062 & 0.052 & 0.055 \\
\cline { 2 - 8 } & 0.80 & 0.011 & 0.011 & 0.016 & 0.044 & 0.057 & 0.059 \\
\cline { 2 - 8 } & 0.85 & 0.009 & 0.008 & $0.019^{*}$ & 0.052 & 0.050 & $0.064^{*}$ \\
\cline { 2 - 8 } & 0.90 & 0.007 & 0.007 & $0.019^{*}$ & 0.048 & 0.046 & $0.064^{*}$ \\
\cline { 2 - 8 } & 0.95 & 0.009 & 0.008 & $0.020^{*}$ & 0.055 & 0.045 & $0.065^{*}$ \\
\hline
\end{tabular}


Table 3: Probabilities of rejection when $H_{0}$ is true $\left(\mu_{x}=\mu_{y}\right)$ of the test statistics Wilcoxon matched Pairs signed-ranks test, Paired sample t-test and $t_{b_{0}^{*}}^{R C M}$, sample size $\mathrm{n}=30, r_{x y}$ be varied from 0.10 to $0.95, \alpha=0.01$ and $\alpha=0.05$.

\begin{tabular}{|c|c|c|c|c|c|c|c|}
\hline $\mathrm{n}$ & $r_{x y}$ & \multicolumn{3}{|c|}{$\alpha=0.01$} & \multicolumn{3}{c|}{$\alpha=0.05$} \\
\cline { 3 - 8 } & & $\mathrm{W}$ & Paired & $t_{b_{0}^{*}}^{R C M}$ & $\mathrm{~W}$ & Paired & $t_{b_{0}^{*}}^{R C M}$ \\
\hline 30 & 0.10 & 0.013 & 0.014 & 0.011 & 0.047 & 0.055 & 0.061 \\
\cline { 2 - 8 } & 0.15 & 0.009 & 0.011 & 0.016 & 0.051 & 0.057 & 0.060 \\
\cline { 2 - 8 } & 0.20 & 0.016 & 0.004 & 0.015 & 0.044 & 0.049 & 0.057 \\
\cline { 2 - 8 } & 0.25 & 0.005 & 0.010 & 0.009 & 0.038 & 0.044 & 0.062 \\
\cline { 2 - 8 } & 0.30 & 0.009 & 0.012 & 0.009 & 0.051 & 0.054 & 0.051 \\
\cline { 2 - 8 } & 0.35 & 0.012 & 0.010 & 0.013 & 0.052 & 0.050 & 0.062 \\
\hline & 0.40 & 0.012 & 0.006 & 0.012 & 0.054 & 0.045 & 0.054 \\
\hline & 0.45 & 0.008 & 0.007 & 0.011 & 0.049 & 0.049 & 0.055 \\
\cline { 2 - 8 } & 0.50 & 0.006 & 0.007 & 0.008 & 0.051 & 0.037 & 0.049 \\
\cline { 2 - 8 } & 0.55 & 0.010 & 0.011 & 0.011 & 0.063 & 0.053 & 0.039 \\
\cline { 2 - 8 } & 0.60 & 0.009 & 0.005 & 0.012 & 0.042 & 0.051 & 0.050 \\
\cline { 2 - 8 } & 0.65 & 0.009 & 0.009 & 0.013 & 0.053 & 0.057 & 0.063 \\
\cline { 2 - 8 } & 0.70 & 0.013 & 0.008 & 0.011 & 0.048 & 0.057 & 0.049 \\
\cline { 2 - 8 } & 0.75 & 0.010 & 0.014 & 0.011 & 0.050 & 0.055 & 0.061 \\
\cline { 2 - 8 } & 0.80 & 0.015 & 0.009 & 0.013 & 0.048 & 0.047 & $0.064^{*}$ \\
\cline { 2 - 8 } & 0.85 & 0.008 & 0.009 & $0.019^{*}$ & 0.042 & 0.056 & $0.064^{*}$ \\
\cline { 2 - 8 } & 0.90 & 0.006 & 0.015 & $0.019^{*}$ & 0.048 & 0.048 & $0.065^{*}$ \\
\cline { 2 - 8 } & 0.95 & 0.009 & 0.010 & $0.020^{*}$ & 0.044 & 0.046 & $0.065^{*}$ \\
\hline
\end{tabular}


Table 4: Probabilities of rejection when $H_{0}$ is true $\left(\mu_{x}=\mu_{y}\right)$ of the test statistics Wilcoxon matched Pairs signed-ranks test, Paired sample t-test and $t_{b_{0}^{*}}^{R C M}$, sample size $\mathrm{n}=40, r_{x y}$ be varied from 0.10 to $0.95, \alpha=0.01$ and $\alpha=0.05$.

\begin{tabular}{|c|c|c|c|c|c|c|c|}
\hline $\mathrm{n}$ & $r_{x y}$ & \multicolumn{3}{|c|}{$\alpha=0.01$} & \multicolumn{3}{c|}{$\alpha=0.05$} \\
\cline { 3 - 8 } & & $\mathrm{W}$ & Paired & $t_{b_{0}^{*}}^{R C M}$ & $\mathrm{~W}$ & Paired & $t_{b_{0}^{*}}^{R C M}$ \\
\hline 40 & 0.10 & 0.006 & 0.015 & 0.013 & 0.053 & 0.049 & 0.059 \\
\cline { 2 - 8 } & 0.15 & 0.008 & 0.015 & 0.012 & 0.052 & 0.048 & 0.054 \\
\cline { 2 - 8 } & 0.20 & 0.011 & 0.014 & 0.010 & 0.046 & 0.059 & 0.062 \\
\cline { 2 - 8 } & 0.25 & 0.007 & 0.007 & 0.015 & 0.047 & 0.051 & 0.048 \\
\cline { 2 - 8 } & 0.30 & 0.011 & 0.015 & 0.016 & 0.053 & 0.048 & 0.061 \\
\cline { 2 - 8 } & 0.35 & 0.007 & 0.004 & 0.015 & 0.050 & 0.043 & 0.052 \\
\cline { 2 - 8 } & 0.40 & 0.011 & 0.008 & 0.010 & 0.043 & 0.046 & 0.049 \\
\cline { 2 - 8 } & 0.45 & 0.007 & 0.013 & 0.012 & 0.053 & 0.055 & 0.054 \\
\cline { 2 - 8 } & 0.50 & 0.014 & 0.006 & 0.008 & 0.048 & 0.051 & 0.061 \\
\cline { 2 - 8 } & 0.55 & 0.004 & 0.017 & 0.008 & 0.049 & 0.043 & 0.044 \\
\cline { 2 - 8 } & 0.60 & 0.008 & 0.005 & 0.012 & 0.049 & 0.056 & 0.051 \\
\cline { 2 - 8 } & 0.65 & 0.014 & 0.010 & 0.009 & 0.043 & 0.056 & 0.056 \\
\cline { 2 - 8 } & 0.70 & 0.008 & 0.008 & 0.015 & 0.047 & 0.059 & 0.057 \\
\cline { 2 - 8 } & 0.75 & 0.006 & 0.009 & 0.013 & 0.047 & 0.051 & 0.063 \\
\cline { 2 - 8 } & 0.80 & 0.012 & 0.008 & $0.019^{*}$ & 0.051 & 0.054 & $0.064^{*}$ \\
\cline { 2 - 8 } & 0.85 & 0.011 & 0.013 & $0.019^{*}$ & 0.049 & 0.055 & $0.064^{*}$ \\
\cline { 2 - 8 } & 0.90 & 0.007 & 0.012 & $0.020^{*}$ & 0.042 & 0.051 & $0.065^{*}$ \\
\cline { 2 - 8 } & 0.95 & 0.013 & 0.010 & $0.020^{*}$ & 0.035 & 0.048 & $0.065^{*}$ \\
\hline
\end{tabular}


Table 5: Probabilities of rejection when $H_{0}$ is true $\left(\mu_{x}=\mu_{y}\right)$ of the test statistics Wilcoxon matched Pairs signed-ranks test, Paired sample t-test and $t_{b_{0}^{*}}^{R C M}$, sample size $\mathrm{n}=50, r_{x y}$ be varied from 0.10 to $0.95, \alpha=0.01$ and $\alpha=0.05$.

\begin{tabular}{|c|c|c|c|c|c|c|c|}
\hline $\mathrm{n}$ & $r_{x y}$ & \multicolumn{3}{|c|}{$\alpha=0.01$} & \multicolumn{3}{c|}{$\alpha=0.05$} \\
\cline { 3 - 8 } & & $\mathrm{W}$ & Paired & $t_{b_{0}^{*}}^{R C M}$ & $\mathrm{~W}$ & Paired & $t_{b_{0}^{*}}^{R C M}$ \\
\hline 50 & 0.10 & 0.011 & 0.009 & 0.013 & 0.047 & 0.042 & 0.059 \\
\cline { 2 - 8 } & 0.15 & 0.005 & 0.010 & 0.016 & 0.047 & 0.041 & 0.060 \\
\cline { 2 - 8 } & 0.20 & 0.009 & 0.013 & 0.011 & 0.047 & 0.052 & 0.050 \\
\cline { 2 - 8 } & 0.25 & 0.013 & 0.011 & 0.016 & 0.041 & 0.044 & 0.044 \\
\cline { 2 - 8 } & 0.30 & 0.010 & 0.015 & 0.008 & 0.040 & 0.054 & 0.055 \\
\cline { 2 - 8 } & 0.35 & 0.009 & 0.008 & 0.011 & 0.041 & 0.045 & 0.052 \\
\hline & 0.40 & 0.009 & 0.012 & 0.009 & 0.044 & 0.049 & 0.051 \\
\hline & 0.45 & 0.008 & 0.010 & 0.009 & 0.052 & 0.042 & 0.049 \\
\cline { 2 - 8 } & 0.50 & 0.012 & 0.007 & 0.008 & 0.052 & 0.058 & 0.042 \\
\cline { 2 - 8 } & 0.55 & 0.009 & 0.011 & 0.014 & 0.047 & 0.046 & 0.047 \\
\cline { 2 - 8 } & 0.60 & 0.015 & 0.010 & 0.006 & 0.063 & 0.048 & 0.062 \\
\cline { 2 - 8 } & 0.65 & 0.008 & 0.007 & 0.011 & 0.051 & 0.047 & 0.042 \\
\cline { 2 - 8 } & 0.70 & 0.007 & 0.013 & 0.016 & 0.054 & 0.046 & 0.050 \\
\cline { 2 - 8 } & 0.75 & 0.010 & 0.010 & 0.008 & 0.042 & 0.048 & 0.062 \\
\cline { 2 - 8 } & 0.80 & 0.009 & 0.010 & $0.019^{*}$ & 0.037 & 0.063 & $0.064^{*}$ \\
\cline { 2 - 8 } & 0.85 & 0.006 & 0.016 & $0.019^{*}$ & 0.048 & 0.053 & $0.064^{*}$ \\
\cline { 2 - 8 } & 0.90 & 0.015 & 0.011 & $0.019^{*}$ & 0.044 & 0.061 & $0.064^{*}$ \\
\cline { 2 - 8 } & 0.95 & 0.013 & 0.013 & $0.020^{*}$ & 0.051 & 0.048 & $0.065^{*}$ \\
\hline
\end{tabular}


Table 6: Probabilities of rejection when $H_{0}$ is not true $\left(\mu_{y}=1.3 \mu_{x}\right)$ of the test statistics Wilcoxon matched pairs signed-ranks test, Paired sample t-test and $t_{b_{0}^{*}}^{R C M}$, sample size $\mathrm{n}=10$, and $r_{x y}$ be varied from 0.10 to $0.95, \alpha=0.01$ and $\alpha=0.05$.

\begin{tabular}{|c|c|c|c|c|c|c|c|}
\hline \multirow[t]{2}{*}{$\mathrm{n}$} & \multirow[t]{2}{*}{$r_{x y}$} & \multicolumn{3}{|c|}{$\alpha=0.01$} & \multicolumn{3}{|c|}{$\alpha=0.05$} \\
\hline & & $\bar{W}$ & Paired & $t_{b_{0}^{*}}^{R C M}$ & $\bar{W}$ & Paired & $t_{b_{0}^{*}}^{R C M}$ \\
\hline \multirow[t]{18}{*}{10} & 0.10 & 0.084 & 0.083 & 0.414 & 0.218 & 0.260 & 0.739 \\
\hline & 0.15 & 0.073 & 0.076 & 0.419 & 0.243 & 0.279 & 0.735 \\
\hline & 0.20 & 0.088 & 0.092 & 0.443 & 0.245 & 0.266 & 0.743 \\
\hline & 0.25 & 0.096 & 0.085 & 0.448 & 0.272 & 0.317 & 0.772 \\
\hline & 0.30 & 0.097 & 0.106 & 0.456 & 0.293 & 0.290 & 0.789 \\
\hline & 0.35 & 0.106 & 0.108 & 0.486 & 0.309 & 0.302 & 0.792 \\
\hline & 0.40 & 0.113 & 0.116 & 0.500 & 0.304 & 0.312 & 0.813 \\
\hline & 0.45 & 0.110 & 0.119 & 0.525 & 0.308 & 0.331 & 0.840 \\
\hline & 0.50 & 0.130 & 0.132 & 0.569 & 0.343 & 0.333 & 0.871 \\
\hline & 0.55 & 0.115 & 0.117 & 0.606 & 0.351 & 0.372 & 0.874 \\
\hline & 0.60 & 0.152 & 0.161 & 0.643 & 0.357 & 0.381 & 0.907 \\
\hline & 0.65 & 0.178 & 0.164 & 0.737 & 0.392 & 0.418 & 0.908 \\
\hline & 0.70 & 0.165 & 0.180 & 0.764 & 0.442 & 0.442 & 0.952 \\
\hline & 0.75 & 0.188 & 0.197 & 0.801 & 0.462 & 0.467 & 0.977 \\
\hline & 0.80 & 0.216 & 0.223 & 0.889 & 0.490 & 0.529 & 0.987 \\
\hline & 0.85 & 0.248 & 0.267 & 0.965 & 0.524 & 0.562 & 0.996 \\
\hline & 0.90 & 0.303 & 0.344 & 0.992 & 0.580 & 0.602 & 0.998 \\
\hline & 0.95 & 0.349 & 0.370 & 0.999 & 0.682 & 0.688 & 1.000 \\
\hline
\end{tabular}


Table 7: Probabilities of rejection when $H_{0}$ is not true $\left(\mu_{y}=1.3 \mu_{x}\right)$ of the test statistics Wilcoxon matched pairs signed-ranks test, Paired sample t-test and $t_{b_{0}^{*}}^{R C M}$, sample size $\mathrm{n}=20$, and $r_{x y}$ be varied from 0.10 to $0.95, \alpha=0.01$ and $\alpha=0.05$.

\begin{tabular}{|c|c|c|c|c|c|c|c|}
\hline \multirow[t]{2}{*}{$\mathrm{n}$} & \multirow[t]{2}{*}{$r_{x y}$} & \multicolumn{3}{|c|}{$\alpha=0.01$} & \multicolumn{3}{|c|}{$\alpha=0.05$} \\
\hline & & $\bar{W}$ & Paired & $t_{b_{0}^{*}}^{R C M}$ & W & Paired & $t_{b_{0}^{*}}^{R C M}$ \\
\hline \multirow[t]{18}{*}{20} & 0.10 & 0.199 & 0.246 & 0.880 & 0.447 & 0.456 & 0.986 \\
\hline & 0.15 & 0.239 & 0.255 & 0.900 & 0.475 & 0.480 & 0.982 \\
\hline & 0.20 & 0.231 & 0.231 & 0.910 & 0.466 & 0.491 & 0.981 \\
\hline & 0.25 & 0.254 & 0.256 & 0.914 & 0.487 & 0.488 & 0.984 \\
\hline & 0.30 & 0.262 & 0.294 & 0.920 & 0.521 & 0.542 & 0.983 \\
\hline & 0.35 & 0.291 & 0.335 & 0.927 & 0.550 & 0.576 & 0.993 \\
\hline & 0.40 & 0.281 & 0.310 & 0.945 & 0.588 & 0.599 & 0.990 \\
\hline & 0.45 & 0.342 & 0.357 & 0.951 & 0.619 & 0.624 & 0.995 \\
\hline & 0.50 & 0.330 & 0.373 & 0.970 & 0.626 & 0.631 & 0.997 \\
\hline & 0.55 & 0.378 & 0.401 & 0.979 & 0.662 & 0.668 & 0.998 \\
\hline & 0.60 & 0.431 & 0.430 & 0.993 & 0.671 & 0.684 & 0.999 \\
\hline & 0.65 & 0.449 & 0.466 & 0.993 & 0.709 & 0.744 & 1.000 \\
\hline & 0.70 & 0.478 & 0.511 & 0.994 & 0.770 & 0.779 & 1.000 \\
\hline & 0.75 & 0.568 & 0.575 & 0.998 & 0.777 & 0.791 & 1.000 \\
\hline & 0.80 & 0.607 & 0.653 & 1.000 & 0.835 & 0.846 & 1.000 \\
\hline & 0.85 & 0.667 & 0.698 & 1.000 & 0.867 & 0.874 & 1.000 \\
\hline & 0.90 & 0.730 & 0.749 & 1.000 & 0.896 & 0.918 & 1.000 \\
\hline & 0.95 & 0.812 & 0.830 & 1.000 & 0.948 & 0.966 & 1.000 \\
\hline
\end{tabular}


Table 8: Probabilities of rejection when $H_{0}$ is not true $\left(\mu_{y}=1.3 \mu_{x}\right)$ of the test statistics Wilcoxon matched pairs signed-ranks test, Paired sample t-test and $t_{b_{0}^{*}}^{R C M}$, sample size $\mathrm{n}=30$, and $r_{x y}$ be varied from 0.10 to $0.95, \alpha=0.01$ and $\alpha=0.05$.

\begin{tabular}{|c|c|c|c|c|c|c|c|}
\hline \multirow[t]{2}{*}{$\mathrm{n}$} & \multirow[t]{2}{*}{$r_{x y}$} & \multicolumn{3}{|c|}{$\alpha=0.01$} & \multicolumn{3}{|c|}{$\alpha=0.05$} \\
\hline & & W & Paired & $t_{b_{0}^{*}}^{R C M}$ & $\mathrm{~W}$ & Paired & $t_{b_{0}^{*}}^{R C M}$ \\
\hline \multirow[t]{18}{*}{30} & 0.10 & 0.371 & 0.413 & 0.989 & 0.662 & 0.652 & 0.997 \\
\hline & 0.15 & 0.415 & 0.428 & 0.984 & 0.673 & 0.673 & 0.999 \\
\hline & 0.20 & 0.410 & 0.437 & 0.988 & 0.653 & 0.661 & 0.999 \\
\hline & 0.25 & 0.440 & 0.449 & 0.996 & 0.690 & 0.706 & 0.999 \\
\hline & 0.30 & 0.438 & 0.495 & 0.993 & 0.732 & 0.738 & 1.000 \\
\hline & 0.35 & 0.493 & 0.499 & 0.999 & 0.759 & 0.761 & 1.000 \\
\hline & 0.40 & 0.498 & 0.520 & 0.999 & 0.781 & 0.788 & 1.000 \\
\hline & 0.45 & 0.528 & 0.545 & 0.999 & 0.789 & 0.799 & 1.000 \\
\hline & 0.50 & 0.598 & 0.596 & 0.999 & 0.839 & 0.840 & 1.000 \\
\hline & 0.55 & 0.627 & 0.657 & 0.999 & 0.869 & 0.852 & 1.000 \\
\hline & 0.60 & 0.648 & 0.684 & 1.000 & 0.888 & 0.879 & 1.000 \\
\hline & 0.65 & 0.701 & 0.737 & 1.000 & 0.948 & 0.895 & 1.000 \\
\hline & 0.70 & 0.740 & 0.769 & 1.000 & 0.913 & 0.928 & 1.000 \\
\hline & 0.75 & 0.806 & 0.821 & 1.000 & 0.942 & 0.949 & 1.000 \\
\hline & 0.80 & 0.831 & 0.850 & 1.000 & 0.944 & 0.972 & 1.000 \\
\hline & 0.85 & 0.874 & 0.916 & 1.000 & 0.969 & 0.972 & 1.000 \\
\hline & 0.90 & 0.928 & 0.950 & 1.000 & 0.990 & 0.992 & 1.000 \\
\hline & 0.95 & 0.980 & 0.982 & 1.000 & 0.996 & 0.996 & 1.000 \\
\hline
\end{tabular}


Table 9: Probabilities of rejection when $H_{0}$ is not true $\left(\mu_{y}=1.3 \mu_{x}\right)$ of the test statistics Wilcoxon matched pairs signed-ranks test, Paired sample t-test and $t_{b_{0}^{*}}^{R C M}$, sample size $\mathrm{n}=40$, and $r_{x y}$ be varied from 0.10 to $0.95, \alpha=0.01$ and $\alpha=0.05$.

\begin{tabular}{|c|c|c|c|c|c|c|c|}
\hline \multirow[t]{2}{*}{$\mathrm{n}$} & \multirow[t]{2}{*}{$r_{x y}$} & \multicolumn{3}{|c|}{$\alpha=0.01$} & \multicolumn{3}{|c|}{$\alpha=0.05$} \\
\hline & & $\mathrm{W}$ & Paired & $t_{b_{0}^{*}}^{R C M}$ & $\mathrm{~W}$ & Paired & $t_{b_{0}^{*}}^{R C M}$ \\
\hline \multirow[t]{18}{*}{40} & 0.10 & 0.486 & 0.504 & 0.998 & 0.737 & 0.787 & 1.000 \\
\hline & 0.15 & 0.532 & 0.550 & 0.999 & 0.753 & 0.792 & 1.000 \\
\hline & 0.20 & 0.547 & 0.601 & 0.999 & 0.809 & 0.833 & 1.000 \\
\hline & 0.25 & 0.582 & 0.626 & 0.999 & 0.819 & 0.841 & 1.000 \\
\hline & 0.30 & 0.643 & 0.657 & 0.999 & 0.831 & 0.837 & 1.000 \\
\hline & 0.35 & 0.657 & 0.693 & 1.000 & 0.847 & 0.866 & 1.000 \\
\hline & 0.40 & 0.680 & 0.689 & 1.000 & 0.894 & 0.887 & 1.000 \\
\hline & 0.45 & 0.701 & 0.749 & 1.000 & 0.907 & 0.921 & 1.000 \\
\hline & 0.50 & 0.761 & 0.765 & 1.000 & 0.918 & 0.926 & 1.000 \\
\hline & 0.55 & 0.793 & 0.793 & 1.000 & 0.923 & 0.938 & 1.000 \\
\hline & 0.60 & 0.819 & 0.826 & 1.000 & 0.947 & 0.954 & 1.000 \\
\hline & 0.65 & 0.865 & 0.889 & 1.000 & 0.950 & 0.961 & 1.000 \\
\hline & 0.70 & 0.893 & 0.901 & 1.000 & 0.971 & 0.979 & 1.000 \\
\hline & 0.75 & 0.913 & 0.938 & 1.000 & 0.986 & 0.984 & 1.000 \\
\hline & 0.80 & 0.949 & 0.953 & 1.000 & 0.992 & 0.995 & 1.000 \\
\hline & 0.85 & 0.957 & 0.967 & 1.000 & 0.994 & 0.996 & 1.000 \\
\hline & 0.90 & 0.981 & 0.986 & 1.000 & 0.994 & 0.997 & 1.000 \\
\hline & 0.95 & 0.992 & 0.996 & 1.000 & 0.998 & 1.000 & 1.000 \\
\hline
\end{tabular}


Table 10: Probabilities of rejection when $H_{0}$ is not true $\left(\mu_{y}=1.3 \mu_{x}\right)$ of the test statistics Wilcoxon matched pairs signed-ranks test, Paired sample t-test and $t_{b_{0}^{*}}^{R C M}$, sample size $\mathrm{n}=50$, and $r_{x y}$ be varied from 0.10 to $0.95, \alpha=0.01$ and $\alpha=0.05$.

\begin{tabular}{|c|c|c|c|c|c|c|c|}
\hline \multirow[t]{2}{*}{$\mathrm{n}$} & \multirow[t]{2}{*}{$r_{x y}$} & \multicolumn{3}{|c|}{$\alpha=0.01$} & \multicolumn{3}{|c|}{$\alpha=0.05$} \\
\hline & & $\bar{W}$ & Paired & $t_{b_{0}^{*}}^{R C M}$ & W & Paired & $t_{b_{0}^{*}}^{R C M}$ \\
\hline \multirow[t]{18}{*}{50} & 0.10 & 0.615 & 0.696 & 0.999 & 0.846 & 0.871 & 1.000 \\
\hline & 0.15 & 0.640 & 0.688 & 1.000 & 0.859 & 0.881 & 1.000 \\
\hline & 0.20 & 0.702 & 0.714 & 1.000 & 0.892 & 0.898 & 1.000 \\
\hline & 0.25 & 0.732 & 0.768 & 1.000 & 0.888 & 0.940 & 1.000 \\
\hline & 0.30 & 0.761 & 0.765 & 1.000 & 0.910 & 0.910 & 1.000 \\
\hline & 0.35 & 0.760 & 0.819 & 1.000 & 0.915 & 0.940 & 1.000 \\
\hline & 0.40 & 0.812 & 0.817 & 1.000 & 0.945 & 0.947 & 1.000 \\
\hline & 0.45 & 0.830 & 0.873 & 1.000 & 0.944 & 0.946 & 1.000 \\
\hline & 0.50 & 0.852 & 0.865 & 1.000 & 0.960 & 0.963 & 1.000 \\
\hline & 0.55 & 0.885 & 0.892 & 1.000 & 0.973 & 83 & 1.000 \\
\hline & 0.60 & 0.914 & 0.932 & 1.000 & 0.976 & 0.982 & 1.000 \\
\hline & 0.65 & 0.932 & 0.933 & 1.000 & 0.988 & 0.992 & 1.000 \\
\hline & 0.70 & 0.941 & 0.953 & 1.000 & 0.989 & 0.994 & 1.000 \\
\hline & 0.75 & 0.982 & 0.976 & 1.000 & 0.998 & 0.998 & 1.000 \\
\hline & 0.80 & 0.985 & 0.985 & 1.000 & 0.999 & 0.999 & 1.000 \\
\hline & 0.85 & 0.994 & 0.996 & 1.000 & 0.999 & 1.000 & 1.000 \\
\hline & 0.90 & 0.997 & 0.999 & 1.000 & 1.000 & 1.000 & 1.000 \\
\hline & 0.95 & 1.000 & 1.000 & 1.000 & 1.000 & 1.000 & 1.000 \\
\hline
\end{tabular}


Table 11: Probabilities of rejection when $H_{0}$ is not true $\left(\mu_{y}=1.5 \mu_{x}\right)$ of the test statistics Wilcoxon matched pairs signed-ranks test, Paired sample t-test and $t_{b_{0}^{*}}^{R C M}$, sample size $\mathrm{n}=10$, and $r_{x y}$ be varied from 0.10 to $0.95, \alpha=0.01$ and $\alpha=0.05$.

\begin{tabular}{|c|c|c|c|c|c|c|c|}
\hline \multirow[t]{2}{*}{$\mathrm{n}$} & \multirow[t]{2}{*}{$r_{x y}$} & \multicolumn{3}{|c|}{$\alpha=0.01$} & \multicolumn{3}{|c|}{$\alpha=0.05$} \\
\hline & & $\mathrm{W}$ & Paired & $t_{b_{0}^{*}}^{R C M}$ & $\mathrm{~W}$ & Paired & $t_{b_{0}^{*}}^{R C M}$ \\
\hline \multirow[t]{18}{*}{10} & 0.10 & 0.223 & 0.248 & 0.883 & 0.505 & 0.534 & 0.985 \\
\hline & 0.15 & 0.252 & 0.270 & 0.896 & 0.531 & 0.576 & 0.990 \\
\hline & 0.20 & 0.282 & 0.304 & 0.912 & 0.570 & 0.590 & 0.991 \\
\hline & 0.25 & 0.280 & 0.291 & 0.920 & 0.596 & 0.580 & 0.995 \\
\hline & 0.30 & 0.302 & 0.305 & 0.937 & 0.596 & 0.613 & 0.996 \\
\hline & 0.35 & 0.301 & 0.325 & 0.948 & 0.623 & 0.640 & 0.997 \\
\hline & 0.40 & 0.329 & 0.344 & 0.953 & 0.675 & 0.654 & 0.998 \\
\hline & 0.45 & 0.349 & 0.393 & 0.955 & 0.682 & 0.680 & 0.999 \\
\hline & 0.50 & 0.400 & 0.407 & 0.972 & 0.713 & 0.727 & 0.999 \\
\hline & 0.55 & 0.427 & 0.422 & 0.984 & 0.731 & 0.748 & 0.999 \\
\hline & 0.60 & 0.449 & 0.464 & 0.991 & 0.776 & 0.788 & 1.000 \\
\hline & 0.65 & 0.517 & 0.523 & 0.991 & 0.807 & 0.817 & 1.000 \\
\hline & 0.70 & 0.537 & 0.551 & 0.994 & 0.800 & 0.841 & 1.000 \\
\hline & 0.75 & 0.597 & 0.631 & 0.999 & 0.870 & 0.874 & 1.000 \\
\hline & 0.80 & 0.623 & 0.686 & 1.000 & 0.899 & 0.916 & 1.000 \\
\hline & 0.85 & 0.692 & 0.720 & 1.000 & 0.928 & 0.935 & 1.000 \\
\hline & 0.90 & 0.787 & 0.783 & 1.000 & 0.956 & 0.954 & 1.000 \\
\hline & 0.95 & 0.833 & 0.865 & 1.000 & 0.976 & 0.980 & 1.000 \\
\hline
\end{tabular}


Table 12: Probabilities of rejection when $H_{0}$ is not true $\left(\mu_{y}=1.5 \mu_{x}\right)$ of the test statistics Wilcoxon matched pairs signed-ranks test, Paired sample t-test and $t_{b_{0}^{*}}^{R C M}$, sample size $\mathrm{n}=20$, and $r_{x y}$ be varied from 0.10 to $0.95, \alpha=0.01$ and $\alpha=0.05$.

\begin{tabular}{|c|c|c|c|c|c|c|c|}
\hline \multirow[t]{2}{*}{$\mathrm{n}$} & \multirow[t]{2}{*}{$r_{x y}$} & \multicolumn{3}{|c|}{$\alpha=0.01$} & \multicolumn{3}{|c|}{$\alpha=0.05$} \\
\hline & & $\bar{W}$ & Paired & $t_{b_{0}^{*}}^{R C M}$ & $\bar{W}$ & Paired & $t_{b_{0}^{*}}^{R C M}$ \\
\hline \multirow[t]{18}{*}{20} & 0.10 & 0.656 & 0.666 & 0.951 & 0.865 & 0.872 & 1.000 \\
\hline & 0.15 & 0.689 & 0.700 & 0.962 & 0.871 & 0.909 & 1.000 \\
\hline & 0.20 & 0.691 & 0.733 & 0.963 & 0.890 & 0.905 & 1.000 \\
\hline & 0.25 & 0.710 & 0.753 & 0.965 & 0.918 & 0.919 & 1.000 \\
\hline & 0.30 & 0.742 & 0.786 & 0.972 & 0.910 & 0.927 & 1.000 \\
\hline & 0.35 & 0.792 & 0.791 & 0.981 & 0.929 & 0.938 & 1.000 \\
\hline & 0.40 & 0.800 & 0.826 & 0.987 & 0.935 & 0.940 & 1.000 \\
\hline & 0.45 & 0.813 & 0.856 & 0.988 & 0.955 & 0.959 & 1.000 \\
\hline & 0.50 & 0.845 & 0.851 & 0.994 & 0.958 & 0.971 & 1.000 \\
\hline & 0.55 & 0.874 & 0.909 & 0.997 & 0.976 & 0.973 & 1.000 \\
\hline & 0.60 & 0.909 & 0.918 & 0.999 & 0.981 & 0.990 & 1.000 \\
\hline & 0.65 & 0.931 & 0.939 & 0.999 & 0.986 & 0.992 & 1.000 \\
\hline & 0.70 & 0.950 & 0.954 & 1.000 & 0.997 & 0.991 & 1.000 \\
\hline & 0.75 & 0.971 & 0.974 & 1.000 & 0.998 & 0.998 & 1.000 \\
\hline & 0.80 & 0.986 & 0.986 & 1.000 & 0.999 & 1.000 & 1.000 \\
\hline & 0.85 & 0.993 & 0.996 & 1.000 & 1.000 & 1.000 & 1.000 \\
\hline & 0.90 & 0.997 & 0.998 & 1.000 & 1.000 & 1.000 & 1.000 \\
\hline & 0.95 & 1.000 & 1.000 & 1.000 & 1.000 & 1.000 & 1.000 \\
\hline
\end{tabular}


Table 13: Probabilities of rejection when $H_{0}$ is not true $\left(\mu_{y}=1.5 \mu_{x}\right)$ of the test statistics Wilcoxon matched pairs signed-ranks test, Paired sample t-test and $t_{b_{0}^{*}}^{R C M}$, sample size $\mathrm{n}=30$, and $r_{x y}$ be varied from 0.10 to $0.95, \alpha=0.01$ and $\alpha=0.05$.

\begin{tabular}{|c|c|c|c|c|c|c|c|}
\hline $\mathrm{n}$ & $r_{x y}$ & \multicolumn{3}{|c|}{$\alpha=0.01$} & \multicolumn{3}{|c|}{$\alpha=0.05$} \\
\cline { 3 - 8 } & & $\mathrm{W}$ & Paired & $t_{b_{0}^{*}}^{R C M}$ & $\mathrm{~W}$ & Paired & $t_{b_{0}^{*}}^{R C M}$ \\
\hline 30 & 0.10 & 0.875 & 0.871 & 1.000 & 0.958 & 0.971 & 1.000 \\
\cline { 2 - 8 } & 0.15 & 0.884 & 0.915 & 1.000 & 0.971 & 0.980 & 1.000 \\
\cline { 2 - 8 } & 0.20 & 0.904 & 0.910 & 1.000 & 0.978 & 0.978 & 1.000 \\
\cline { 2 - 8 } & 0.25 & 0.912 & 0.935 & 1.000 & 0.984 & 0.986 & 1.000 \\
\cline { 2 - 8 } & 0.30 & 0.936 & 0.946 & 1.000 & 0.986 & 0.990 & 1.000 \\
\cline { 2 - 7 } & 0.35 & 0.955 & 0.951 & 1.000 & 0.994 & 0.992 & 1.000 \\
\hline 0.40 & 0.963 & 0.971 & 1.000 & 0.995 & 0.995 & 1.000 \\
\hline 0.45 & 0.963 & 0.974 & 1.000 & 0.996 & 0.997 & 1.000 \\
\hline 0.50 & 0.979 & 0.985 & 1.000 & 0.997 & 0.998 & 1.000 \\
\hline 0.55 & 0.991 & 0.988 & 1.000 & 0.999 & 0.999 & 1.000 \\
\hline 0.60 & 0.991 & 0.998 & 1.000 & 0.999 & 0.999 & 1.000 \\
\cline { 2 - 8 } & 0.65 & 0.989 & 0.998 & 1.000 & 1.000 & 1.000 & 1.000 \\
\cline { 2 - 7 } & 0.70 & 0.997 & 0.999 & 1.000 & 1.000 & 1.000 & 1.000 \\
\cline { 2 - 7 } & 0.75 & 0.999 & 0.999 & 1.000 & 1.000 & 1.000 & 1.000 \\
\cline { 2 - 7 } & 0.80 & 1.000 & 1.000 & 1.000 & 1.000 & 1.000 & 1.000 \\
\cline { 2 - 7 } & 0.85 & 1.000 & 1.000 & 1.000 & 1.000 & 1.000 & 1.000 \\
\cline { 2 - 7 } & 0.90 & 1.000 & 1.000 & 1.000 & 1.000 & 1.000 & 1.000 \\
\cline { 2 - 7 } & 0.95 & 1.000 & 1.000 & 1.000 & 1.000 & 1.000 & 1.000 \\
\hline
\end{tabular}


Table 14: Probabilities of rejection when $H_{0}$ is not true $\left(\mu_{y}=1.5 \mu_{x}\right)$ of the test statistics Wilcoxon matched pairs signed-ranks test, Paired sample t-test and $t_{b_{0}^{*}}^{R C M}$, sample size $\mathrm{n}=40$, and $r_{x y}$ be varied from 0.10 to $0.95, \alpha=0.01$ and $\alpha=0.05$.

\begin{tabular}{|c|c|c|c|c|c|c|c|}
\hline \multirow[t]{2}{*}{$\mathrm{n}$} & \multirow[t]{2}{*}{$r_{x y}$} & \multicolumn{3}{|c|}{$\alpha=0.01$} & \multicolumn{3}{|c|}{$\alpha=0.05$} \\
\hline & & W & Paired & $t_{b_{0}^{*}}^{R C M}$ & $\mathrm{~W}$ & Paired & $t_{b_{0}^{*}}^{R C M}$ \\
\hline \multirow[t]{18}{*}{40} & 0.10 & 0.963 & 0.976 & 1.000 & 0.994 & 0.994 & 1.000 \\
\hline & 0.15 & 0.975 & 0.971 & 1.000 & 0.997 & 0.997 & 1.000 \\
\hline & 0.20 & 0.977 & 0.980 & 1.000 & 0.998 & 0.998 & 1.000 \\
\hline & 0.25 & 0.980 & 0.983 & 1.000 & 0.988 & 0.998 & 1.000 \\
\hline & 0.30 & 0.992 & 0.992 & 1.000 & 0.999 & 0.999 & 1.000 \\
\hline & 0.35 & 0.991 & 0.997 & 1.000 & 0.999 & 1.000 & 1.000 \\
\hline & 0.40 & 0.995 & 0.996 & 1.000 & 0.999 & 1.000 & 1.000 \\
\hline & 0.45 & 0.995 & 0.997 & 1.000 & 1.000 & 1.000 & 1.000 \\
\hline & 0.50 & 0.997 & 1.000 & 1.000 & 1.000 & 1.000 & 1.000 \\
\hline & 0.55 & 0.999 & 1.000 & 1.000 & 1.000 & 1.000 & 1.000 \\
\hline & 0.60 & 1.000 & 1.000 & 1.000 & 1.000 & 1.000 & 1.000 \\
\hline & 0.65 & 1.000 & 1.000 & 1.000 & 1.000 & 1.000 & 1.000 \\
\hline & 0.70 & 1.000 & 1.000 & 1.000 & 1.000 & 1.000 & 1.000 \\
\hline & 0.75 & 1.000 & 1.000 & 1.000 & 1.000 & 1.000 & 1.000 \\
\hline & 0.80 & 1.000 & 1.000 & 1.000 & 1.000 & 1.000 & 1.000 \\
\hline & 0.85 & 1.000 & 1.000 & 1.000 & 1.000 & 1.000 & 1.000 \\
\hline & 0.90 & 1.000 & 1.000 & 1.000 & 1.000 & 1.000 & 1.000 \\
\hline & 0.95 & 1.000 & 1.000 & 1.000 & 1.000 & 1.000 & 1.000 \\
\hline
\end{tabular}


Table 15: Probabilities of rejection when $H_{0}$ is not true $\left(\mu_{y}=1.5 \mu_{x}\right)$ of the test statistics Wilcoxon matched pairs signed-ranks test, Paired sample t-test and $t_{b_{0}^{*}}^{R C M}$, sample size $\mathrm{n}=50$, and $r_{x y}$ be varied from 0.10 to $0.95, \alpha=0.01$ and $\alpha=0.05$.

\begin{tabular}{|c|c|c|c|c|c|c|c|}
\hline \multirow[t]{2}{*}{$\mathrm{n}$} & \multirow[t]{2}{*}{$r_{x y}$} & \multicolumn{3}{|c|}{$\alpha=0.01$} & \multicolumn{3}{|c|}{$\alpha=0.05$} \\
\hline & & $\mathrm{W}$ & Paired & $t_{b_{0}^{*}}^{R C M}$ & $\mathrm{~W}$ & Paired & $t_{b_{0}^{*}}^{R C M}$ \\
\hline \multirow[t]{18}{*}{50} & 0.10 & 0.991 & 0.991 & 1.000 & 0.999 & 0.999 & 1.000 \\
\hline & 0.15 & 0.992 & 0.996 & 1.000 & 0.999 & 0.999 & 1.000 \\
\hline & 0.20 & 0.994 & 0.996 & 1.000 & 0.999 & 1.000 & 1.000 \\
\hline & 0.25 & 0.998 & 0.997 & 1.000 & 1.000 & 1.000 & 1.000 \\
\hline & 0.30 & 0.998 & 0.999 & 1.000 & 1.000 & 1.000 & 1.000 \\
\hline & 0.35 & 0.999 & 0.999 & 1.000 & 1.000 & 1.000 & 1.000 \\
\hline & 0.40 & 0.999 & 1.000 & 1.000 & 1.000 & 1.000 & 1.000 \\
\hline & 0.45 & 0.999 & 1.000 & 1.000 & 1.000 & 1.000 & 1.000 \\
\hline & 0.50 & 1.000 & 1.000 & 1.000 & 1.000 & 1.000 & 1.000 \\
\hline & 0.55 & 1.000 & 1.000 & 1.000 & 1.000 & 1.000 & 1.000 \\
\hline & 0.60 & 1.000 & 1.000 & 1.000 & 1.000 & 1.000 & 1.000 \\
\hline & 0.65 & 1.000 & 1.000 & 1.000 & 1.000 & 1.000 & 1.000 \\
\hline & 0.70 & 1.000 & 1.000 & 1.000 & 1.000 & 1.000 & 1.000 \\
\hline & 0.75 & 1.000 & 1.000 & 1.000 & 1.000 & 1.000 & 1.000 \\
\hline & 0.80 & 1.000 & 1.000 & 1.000 & 1.000 & 1.000 & 1.000 \\
\hline & 0.85 & 1.000 & 1.000 & 1.000 & 1.000 & 1.000 & 1.000 \\
\hline & 0.90 & 1.000 & 1.000 & 1.000 & 1.000 & 1.000 & 1.000 \\
\hline & 0.95 & 1.000 & 1.000 & 1.000 & 1.000 & 1.000 & 1.000 \\
\hline
\end{tabular}




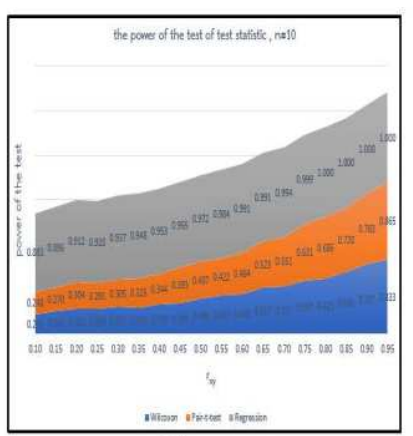

(a)

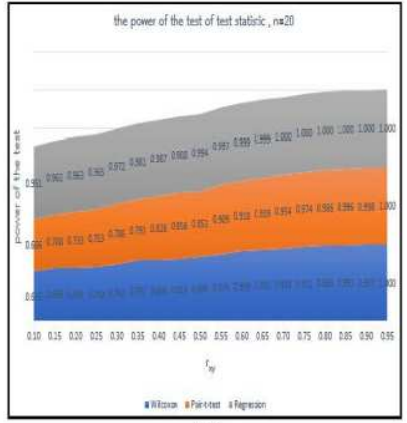

(b)

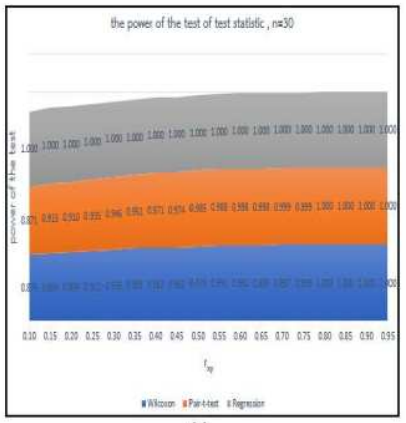

(c)

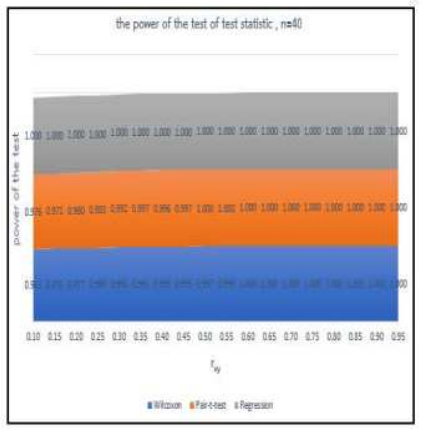

(d)



(e)

Figure 4: Power of the test of test statistics Wilcoxon matched Pairs signed-ranks test, Paired sample t-test, and $t_{b_{0}^{*}}^{R C M}, \mu_{y}=1.5 \mu_{x}$, $\alpha=0.01$, and $r_{x y}$ be varied from 0.10 to 0.95 .(a) $\mathrm{n}=10$,(b) $\mathrm{n}=20,(\mathrm{c})$ $\mathrm{n}=30,(\mathrm{~d}) \mathrm{n}=40$ and (e) $\mathrm{n}=50$. 


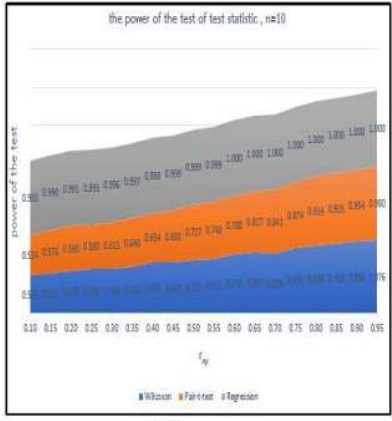

(a)

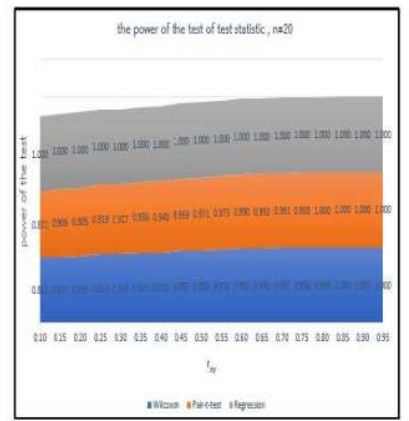

(b)

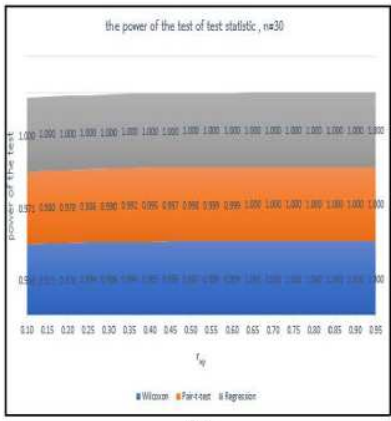

(c)

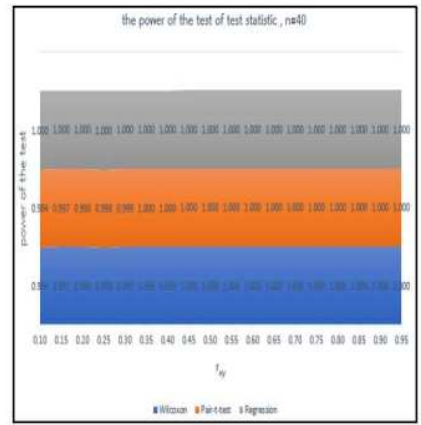

(d)



(e)

Figure 5: Power of the test of test statistics Wilcoxon matched Pairs signed-ranks test, Paired sample t-test, and $t_{b_{0}^{*}}^{R C M}, \mu_{y}=1.5 \mu_{x}$, $\alpha=0.05$, and $r_{x y}$ be varied from 0.10 to 0.95 .(a) $\mathrm{n}=10$,(b) $\mathrm{n}=20,(\mathrm{c})$ $\mathrm{n}=30,(\mathrm{~d}) \mathrm{n}=40$ and (e) $\mathrm{n}=50$.

A similar finding is found when $\mathrm{n}$ is varied from 5 to 100 and the value of $r_{x y}$ is varied from 0.05 to 1.00, as shown in Fig. 6. As regards the power of the test ratio of the Wilcoxon matched-pairs signed-ranks test to the $t_{b_{0}^{*}}^{R C M}$ at the significance level of $\alpha=0.01$, as shown in Fig. 6(a), and at the significance level of $\alpha=0.05$, as shown in Fig. 6(c), it is found that the ratio of the power of the test $\frac{\text { Wilcoxon }}{t_{b_{0}^{*}}^{R C M}}<1$ when $n \leq 45$ and $r \leq 0.45$. That is, the power of the test of the latter is higher than that of the former. In contrast, the power of the test of the two test statistics does not differ when $n>45$ and $r>0.45$ at the significance level of both $\alpha=0.01$ and $\alpha=0.05$. As for the power of the test ratio of the paired sample t-test to the $t_{b_{0}^{*}}^{R C M}$ at the significance level of $\alpha=0.01$, as shown in Fig. 6(b), and at the significance level of $\alpha=0.05$, as shown in Fig. 6(d), it is similarly found that $\frac{\text { Paired sample t-test }}{t_{b_{0}^{R C M}}^{R C M}}<1$ when 


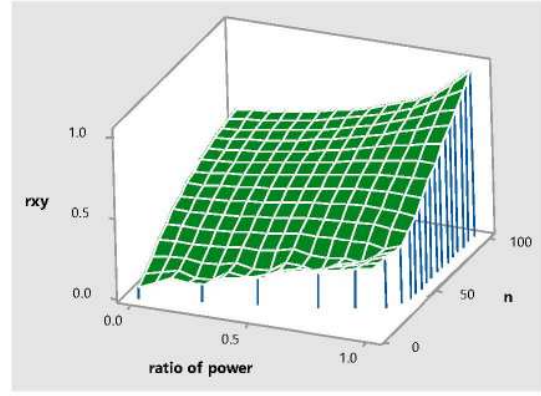

(a)

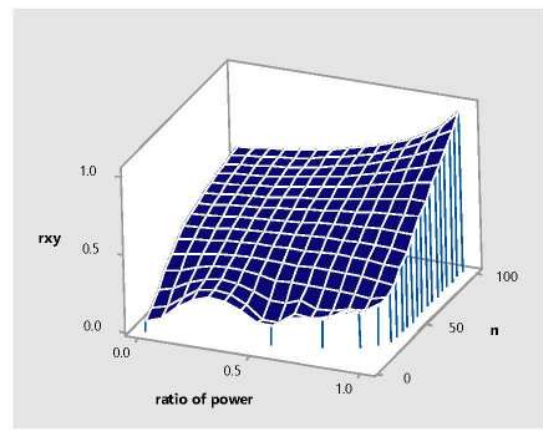

(c)

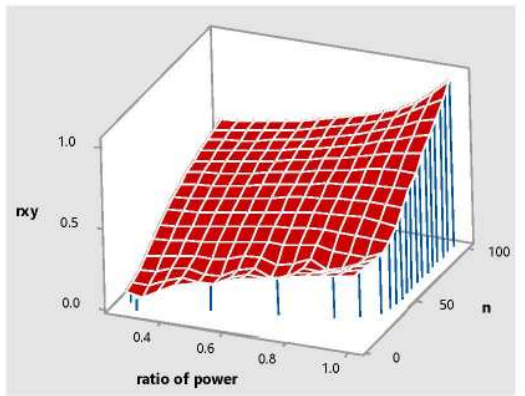

(b)

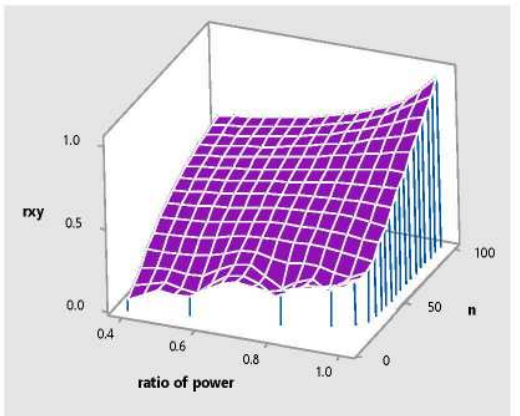

(d)

Figure 6: Comparison the ratio of the power of the test where $\mu_{y}=$ $1.5 \mu_{x}$ (a) $\frac{\text { Wilcoxon }}{t_{b_{0}^{*}}^{R C M}}, \alpha=0.01$.(b) $\frac{\text { Paired sample t-test }}{t_{b_{0}^{*}}^{R C M}}, \alpha=0.01$.(c) $\frac{\text { Wilcoxon }}{t_{b_{0}^{*}}^{R C M}}, \alpha=0.05 .(\mathrm{d}) \frac{\text { Paired sample t-test }}{t_{b_{0}^{*}}^{R C M}}, \alpha=0.05$.

$n \leq 40$ and $r \leq 0.40$. That is, the power of the test of the latter is higher than that of the former. In contrast, there is no difference in the power of the test between the two test statistics when $n>40$ and $r>0.40$ at the significance level of both $\alpha=0.01$ and $\alpha=0.05$.

\section{Conclusion and Discussion}

From the analysis of the efficiency of the Wilcoxon matched-pairs signed-ranks test, the Paired sample t-test, and the $t_{b_{0}^{*}}^{R C M}$, when $\sigma_{\bar{d}}$ of paired sample ttest and $\sigma_{b_{0}^{*}}$ of regression center model are considered, it is found that the value of $\sigma_{b_{0}^{*}}$ is lower than that of $\sigma_{\bar{d}}$, likely due to $\sigma_{\bar{d}}=\sqrt{\frac{\sigma_{y}^{2}+\sigma_{x}^{2}-2 \rho_{x y} \sigma_{x} \sigma_{y}}{n}}$. 
Additionally, $\sigma_{b_{0}^{*}}=\sqrt{\frac{\left(\sigma_{y}^{2}-\rho_{x y}^{2} \sigma_{y}^{2}\right)}{n}}$ causes the value of $t_{b_{0}^{*}}^{R C M}$ to be higher than that of Paired sample t-test, resulting in the former involving a higher chance of hypothesis rejection than the latter. With regards to the ability to control Type I errors, it is found that the Wilcoxon matched-pairs signed-ranks test and the Paired sample t-test are efficient under all scenarios, while the $t_{b_{0}^{*}}^{R C M}$ performs well when the value of $r_{x y}$ is not excessively high, that is, lower than 0.80. In contrast, the $t_{b_{0}^{*}}^{R C M}$ loses its ability to control Type I errors in case the value of $r_{x y}$ goes beyond 0.80. As for the power of the test, it is found on the contrary that the $t_{b_{0}^{*}}^{R C M}$ fares the best under all scenarios, followed by the Paired sample t-test and the Wilcoxon matched-pairs signed-ranks test, respectively. In addition, the power of the test of the $t_{b_{0}^{*}}^{R C M}$ is stronger with an increase in the value of the correlation $r_{x y}$ and the sample sizes. Moreover, when the value of $r_{x y}$, the ratio of the mean difference between the two datasets $\left(\mu_{y}=1.3 \mu_{x}, \mu_{y}=1.5 \mu_{x}\right)$, and increases in sample sizes are compared, it is found that the most influential factor is the second. That is, an increase in the mean difference between the two datasets will lead to such a growth in the power of the test of the three test statistics that it converges. Based on the present findings, it can thus be concluded that actual applications of the three test statistics in testing the difference between two dependent datasets should take account of the following considerations. Generally, the Wilcoxon matched-pairs signed-ranks test and the Paired sample t-test are the test statistics to be opted for in case the emphasis is the ability to control Type I errors. However, the $t_{b_{0}^{*}}^{R C M}$ may be a feasible alternative provided that the correlation coefficient $r_{x y}$ of the two datasets under investigation does not exceed 0.80. More importantly, such a test statistic is the strongest in terms of the power of the test under all scenarios.

\section{Acknowledgement}

The authors gratefully acknowledge the participating in the Faculty of Science and Technology, Rajamangala University of Technology Thanyaburi. We are also thankful to those who could not be mentioned here for their kindness and encouragement.

\section{References}

[1] A. Gelman, and J. Hill, Data Analysis Using Regression and Multilevel/Hierarchical Models, Cambridge University Press (2006). 
[2] D.L. Street, Controlling extraneous variables in experimental research: A research note, Accounting Education, 6, No 2 (1995), 169-188.

[3] D. Torgerson, Designing Randomised Trials in Health, Education and the Social Sciences: An Introduction, Springer (2008).

[4] E.C. Hedberg, and S. Ayers, Controlling extraneous variables in experimental research: The power of a paired t-test with a covariate, Social Science Research, 50 (2015), 277-291.

[5] E. Marsden, and C.J. Torgerson, Single group, pre-and post-test research designs: Some methodological concerns, Oxford Review of Education, 38, No 5 (2012), 583-616.

[6] F.J. Samaniego, Stochastic Modeling and Mathematical Statistics: A Text for Statisticians and Quantitative Scientists, Chapman and Hall/CRC (2014).

[7] H.J. Keselman, R. Cribbie, and B. Holland, Controlling the rate of Type I error over a large set of statistical tests, British Journal of Mathematical and Statistical Psychology, 55, No 1 (2002), 27-39.

[8] J. Hox, and J.K. Roberts, Handbook of Advanced Multilevel Analysis, Psychology Press (2011).

[9] J. Miles, and M. Shevlin, Applying Regression and Correlation: A guide for Students and Researchers, Sage (2001).

[10] P.D. Mehta, Control Variables in Research (2015).

[11] R.H. Myers, and J.S. Milton, A First Course in the Theory of Linear Statistical Models, Kent Publishing Company (1991).

[12] R.W. Robins, R.C. Fraley, and R.F. Krueger, Handbook of Research Methods in Personality Psychology, Guilford Press (2009).

[13] S.D. Oman, Easily simulated multivariate binary distributions with given positive and negative correlations, Computational Statistics and Data Analysis, 53, No 4 (2009), 999-1005.

[14] T.W. MacFarland, and J.M. Yates, Wilcoxon matched-pairs signed-ranks test, In: Introduction to Nonparametric Statistics for the Biological Sciences Using R, Springer, Cham (2016). 
[15] W.R. Shadish, T.D. Cook, and D.T. Campbell, Experimental and QuasiExperimental Designs for Generalized Causal Inference, Boston, Houghton Mifflin (2002). 
